\title{
Spatial Domain Approach for Articular Cartilage Segmentation of X-Ray Image
}

\author{
Vijayakumari G. \\ Dept. of CSE \\ SLN College of Engineering \\ Raichur, VTU, Belagavi \\ Karnataka, India
}

\author{
Ganga Holi \\ Dept. of ISE \\ Global Academy of Technology \\ Bengaluru, VTU, Belagavi \\ Karnataka, India
}

\begin{abstract}
Cartilage is a protective tissue which covers the knee bone joints and also allows bones to glide easily and prevents friction between bones. Cartilage slowly degenerates due to age, wear and tear and obese causing joint inflammation which is referred as Osteoarthritis (OA). There is need to estimate the cartilage thickness to find out the severity of the OA. In this paper, image processing algorithms are applied to Knee X-ray images to segment the cartilage portion and to find out the thickness. Spatial domain techniques of image processing such as pre-processing, contrast enhancement, segmentation, post processing methods are employed to preprocess the image and segment the cartilage portion. The proposed method works well on the images collected from local hospitals and segments the cartilage portion.
\end{abstract}

\section{Keywords}

Osteoarthritis(OA), Adaptive Histogram Equalization, Thresholding, GLCM, Morphological Operations.

\section{INTRODUCTION}

Osteoarthritis is the type of common arthritis which affects the bone joint which slowly reduces the size of the cartilage and restricts bone mobility freely. OA has been considered as a major musculoskeletal chronic health state which causes irreversible and progressive damage in articular cartilage tissue. Due to degeneration of the cartilage, mobility causes friction within the bone joints causing severe pain [1] in the joint. OA commonly occurs in the bone joints, mainly at joints which connect thumb and hand, fingertip, near the wrist, knee joint, toes, backbone (spine), and hip. There are various modalities are available to capture the cartilage image using $\mathrm{X}$-ray, MRI \& CT methods. When compared to X-ray imaging MRI and CT will provide best results but these are more expensive. In this paper, spatial domain approaches are proposed to detect cartilage thickness by calculating accurate width of cartilage tissue along with GLCM which provides the textural features of an image.

The existing systems for recognition of OA are i) Joint Space Width technique ii) Region of interest iii) KellegrenLawrence method and iv) Edge detection algorithm. The limitations of existing systems are i) boundary appear as discontinuous, ii) exact detection of knee regions, iii) accuracy is low and iv) losing of edge information. Figure 1 shows the anatomy of the human knee and the surrounding terms. Due to age factor or wear and tear of knee, when the quantity of the meniscus (which will be in liquid form) get reduced, leading friction and wearing out of cartilage in turn causing pain in knee joint. Cartilage thickness can be calculated by measuring the gap between the upper and lower portion of the cartilage. Accurate measurement of the width is an important method to identify the severity of the OA [2]. '
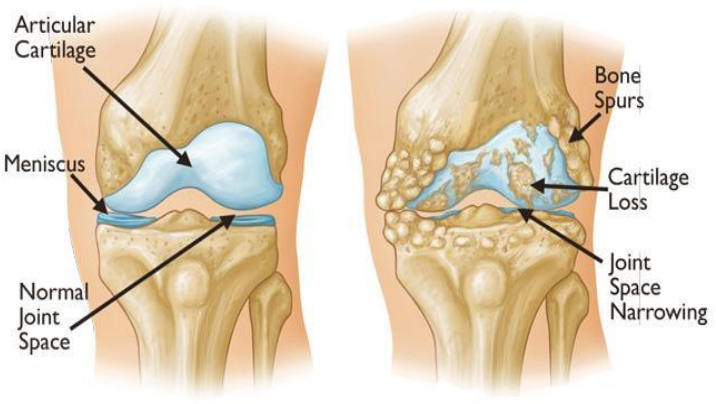

Figure. 1 Anatomy of Human Knee

Image Courtesy: https://orthoinfo.aaos.org/en/diseasesconditions/arthritis-of-the-knee.

This paper is organized into 5 sections: section 1 describes the introduction of the Knee Osteoarthritis, section 2 deals with the related work on automatic segmentation of cartilage. Section 3 discusses the proposed methodology. Section 4 deals with experimental results and discussion and the conclusion is given in section 5 .

\section{RELATED WORK}

Many authors have proposed several strategies for the detection and analysis of $\mathrm{OA}$ images in various forms knee pictures like X-ray, MRI, \& CT etc. Image segmentation plays very important role in most medical imaging applications. Jessie Thomson et al. [3] , have used fully automated computer aided system that identifies the outlines of the knee joint bones. The authors have used Random Forest Regression Voting Constrained Local Model (RFCLM) and Statistical shape model for automatic analysis. Lior Shamir et al.[4] have proposed machine-controlled method for detection of degenerative joint disease using knee $\mathrm{X}$-ray image. In this paper, the Kellgren-Lawrence classification method has been used for detection, which provides different stages of OA and helps to detect the severity. The algorithm was designed to train manually to demonstrate the first four conditions of KL. Initially, the input X-ray image is analyzed by identifying set content descriptors and transforms the image, which is more efficient for the detection of OA and assigns values to those image features by using Fisher scores. Shepherd et al.[5] have implemented Statistical analysis method to compare the thickness of the cartilage of lower limb joints and surface of the individual joints of two different groups of participants.

Mahima Shanker Pandey et al. [6] have proposed an automated computer-assisted technique to identify OA using 
knee X-ray images based on joint space width (JSW). In this method, image contrast has been enhanced by implementing Gaussian low pass filter (GLPF) and contrast stretching method to get better quality output image. Euclidean distance equation is applied to measure the joint space width, which is then compared with the normal value. In this paper, overall 100 images are considered in which the success rate was up to $85 \%$. Samir K. Bandyopadhyay [7] has proposed an algorithm to detect edges in Knee X-ray images to identify osteoarthritis. In this method, few features like thickness and distance between femur, tibia and patella cartilage were computed. This method provides an efficient result and concluded as the efficient method to implement in noisy and blur images. Prafull Sharma et al. [8] have used a new approach in the segmentation of X-ray images called discrete step algorithm which provides a quick and efficient method to analyze the abnormalities and problems associated with bone structures. It gives an easy step by step approach in X-Ray image segmentation, which gives better bone segmentation results comparatively with Otsu's and Watershed segmentation methods.

Subromoniam M et al. [9] have implemented a computeraided diagnosis to detect osteoarthritis by using X-ray images. They employed Haralick feature extraction method for computation and SVM classifier used to classify the images. In this technique, the authors have obtained 99\% accuracy while diagnosing bone disorder which is caused by osteoarthritis.

Tati L.Mengko et al. [10] have implemented a machine vision system for detection of OA. In this image segmentation method, edge detection method has been used to identify the region of joint space. In order to classify normal \& OA affected X-ray images, neural network is used and classified. Later results are evaluated by comparing manually detected method. The proposed method produced result with sensitivity of $50 \%, 91.84 \%$ and $100 \%$ specificity \& positive predictive value respectively. Lior Shamir [10] has proposed a computer-based image analysis method to identify correlations within X-ray Images \& clinical indicators. In this approach, WND-CHRM algorithm has been used along with few other feature extraction methods like Haralick, Tamura, and Statistical. The obtained results are classified using the Weighted Nearest Neighbor technique in which a satisfactory result have been obtained by comparing X-ray Images \& clinical indicators [11].

In [12], authors have used Active contour segmentation technique to segment the portion/part of the knee X-ray image to diagnosis the disease. The numerous features like Haralick, Statistical, First four moments, Texture, and Shape are computed and classified using a Random Forest classifier.

An automated technique for the visualization and mapping of articular cartilage in magnetic resonance (MR) images has been proposed in [13]. Authors have developed and validated method for automated segmentation and thickness mapping of articular cartilage from three-dimensional (3-D) gradient-echo MR images of the knee.

Dian Pratiwi et al. [14] have used artificial neural with back propagation method for measuring the severity of Osteoarthritis disease. The method is divided into three steps, image processing, feature extraction and classification using artificial neural network process.

Emuoyibofarhe O.Justice [15] proposed fuzzy-based system to determine the severity of knee osteoarthritis. The fuzzy logic controller is used on four parameters such as knee pain, stiffness, crepitus \& age to determine the severity. The results show that the parameter "knee pain" \& "stiffness" are directly proportional to variable "severity-level", which will determine the level of severity of knee osteoarthritis. This paper reports the development of a fuzzy system that allows determining the level of severity of knee osteoarthritis.

Another method [16] based on the image segmentation and mathematical morphological operators have implemented to extract the border of cartilage by covering the boundary of cartilage.

From the above literature, it is evident that many of researchers have done significant amount of work on Knee Xray images to detect and classify OA, by implementing different approaches and obtained efficient results with their datasets. Many researchers are working on improving the existing method to get more accurate results and better understanding of the severity. The proposed method is one such approach in enhancing and segmenting the cartilage portion and calculates the width. The proposed methodology is explained in the next section.

\section{PROPOSED METHOD}

\subsection{Dataset Collection}

The Knee X-ray images of OA patients are collected from Balanku hospital, Raichur, Karnataka. The dataset nearly containing 100 images of which $60 \%$ are from female patients. The ages of the patients varying from age 45 to 75 with average age of 60 .

\subsection{Preprocessing}

The collected Knee X-ray images are converted into gray scale image and used for further processing. The captured Xray image usually needs pre-processing as X-ray images usually contains low intensity and uneven illumination. Gaussian smoothing is used to smooth the image. In 2-D, the circular symmetric Gaussian has the form given below:

$$
f(x, y)=\frac{1}{2 \pi \sigma^{2}} e^{\frac{x^{2}+y^{2}}{2 \sigma^{2}}}
$$

Where $x$ is the distance from the origin in the horizontal axis, $y$ is the distance from the origin in the vertical axis, and $\sigma$ is the standard deviation of the Gaussian distribution. Adaptive Histogram Equalization (AHE) is applied to improve the image contrast. The filtered image is enhanced using AHE which operates on the small region (tiles), rather than the entire image. Contrast enhancement is performed on each tile depending on the local intensity variation.

\subsection{Thresholding}

Thresholding is one of the most simple segmentation techniques to segment the image into multiple segments. It uses the threshold value calculated based on various parameters and segments the gray scale pixels into classes depends on how many threshold values are used. If the threshold value is one, then image will be segmented into two classes/segments. If the thresholding values used are more than one, then the image will be segmented into (thresholding values +1 ) groups/classes. If image is segmented into class with one thresholding value, then it is referred as binarization and is given by equation (2), the resultant image will be binary image if we use 0 and 1 values for the segmented image intensity. 


$$
f(x, y)=\left\{\begin{array}{l}
0 \text { if } f(x, y)<t \\
1 \text { if } f(x, y) \geq t
\end{array}\right.
$$

Where $f(x, y)$ represents a gray value and $t$ is the threshold value. If $\mathrm{n}$ more than one thresholding values are used, then segmented image will be having $\mathrm{n}+1$ segment with intensity values $V_{1}, V_{2}, V_{3} \ldots V_{n}$ and is represented by equation (3). $f(x, y)=$

$$
\left\{\begin{array}{cc}
V_{1} & \text { if } f(x, y)<t_{1} \\
V_{2} & \text { if } f(x, y) \geq t_{1} \text { and } f(x, y)<t_{2} \\
V_{3} & \text { if } f(x, y) \geq t_{2} \text { and } f(x, y)<t_{n} \\
\cdot & \cdot \\
V_{n+1} & \cdot \text { if } f(x, y) \geq t_{n}
\end{array}\right.
$$

The proposed technique uses global thresholding Ostu [17] method to find the thresholding value and image is binarized after AHE method.

\subsection{Edge Detection}

Edge detecting an image significantly reduces the amount of data and filters out useless information, while preserving the important structural properties in an image. The binary gradient is a directional change in the intensity or color in an image. Image gradients are accustomed to extracting data from pictures. Gradient images are created from the original image by convolving with a filter, one of the simplest being the Sobel filter. Each constituent of a gradient image measures the modification in the intensity of that very same purpose within the original image, in a given direction. To get the complete vary of direction, gradient pictures within the $\mathrm{x}$ and $\mathrm{y}$ directions are computed.

One of the most common uses of image gradients is in edge detection. After gradient pictures are computed, pixels with massive gradient values become possible edge pixels. The pixels with the biggest gradient values within the direction of the gradient become edge pixels, and edges are also derived within the direction perpendicular to the gradient direction. The magnitude and the direction of the gradient are obtained

by $: \nabla f=\left[\begin{array}{l}g_{x} \\ g_{y}\end{array}\right]=\left[\begin{array}{l}\frac{\delta f}{\delta x} \\ \frac{\delta f}{\delta y}\end{array}\right]$

Where: $\frac{\delta f}{\delta x}$ the derivative with respect to $\mathrm{x}$ (gradient in the $\mathrm{x}$ direction) and $\frac{\delta f}{\delta y}$ the derivative with respect to y (gradient in the y direction).

$$
\theta=\tan ^{-1}\left(\frac{g_{y}}{g_{x}}\right)
$$

The result of binary gradient of a segmented knee X-ray is shown in Fig 7.

The segmentation of the cartilage is performed by active contour method, a region based proposed by Chan-Vese [18]. Well known, powerful and adaptable method to segment the images which are difficult to segment using thresholding and uses gradients. The Chan-Vese algorithm is used in medical image processing.

\subsection{Cartilage thickness Calculation}

Thickness measurement of cartilage tissue is considered as a major standard method in the diagnosis of OA from X-ray image for defining of the disease. The cartilage thickness is defined as the distance between upper and lower cartilage and is computed using hamming distance formula in order to find the region of interest (Osteoarthritis). The formula for Hamming distance is given by:

$0\left|r_{i} \cap s_{k}\right|$

$$
D_{-} H(S \Rightarrow R)=\sum r_{i} \in R \sum s_{k} \neq s_{j}, s_{k} \cap r_{i} \neq
$$

$P=1-\frac{D_{H}(S \Rightarrow R)+D_{H}(R \Rightarrow S)}{2 \times|S|}$

Where $\mathrm{S}$ and $\mathrm{R}$ are the two segmentations, $r i, s k, s j$ are regions belongs to the segmentations $\mathrm{R}$ and $\mathrm{S}$ respectively, and $D_{H}$ is a hamming distance.

The main advantage of the proposed method is the high accuracy rate in detecting Osteoarthritis by using the features such as contrast, correlation, energy, and homogeneity.

\subsection{Texture Features for finding the severity of the cartilage}

Post processing is done using morphological operations dilation. Dilation process bridges the gap between pixels and expands the boundary which is helpful in locating the border of cartilage. The Gray Level Co-occurrence Matrix (GLCM) is a statistical method for extracting textural features of images. GLCM features can be used reflect the overall average for the degree of correlation among pixel pairs in various aspects like: homogeneity, uniformity, energy, entropy, etc. These features are computed using following equations.

- Contrast: Measures the local variations in the gray-level co-occurrence matrix.

$$
\text { Contrast }=\sum_{i, j=0}^{N-1} P_{i j}(i-j)^{2}
$$

- Correlation: Correlation measures the linear dependency of gray levels of neighboring pixels.

$$
\text { Correlation }=\frac{(i-\mu)(j-\mu)}{\sigma^{2}}
$$

- Energy: It is a gray-scale image texture measure of homogeneity changing, reflecting the distribution of image gray-scale uniformity of weight and texture.

$$
\text { Energy }=\sum_{i, j=0}^{N-1}\left(P_{i j}\right)^{2}
$$

- Homogeneity: Measures the closeness of the distribution of elements in the GLCM to the GLCM diagonal.

$$
\text { Homogeneity }=\quad \sum_{i, j=0}^{N-1} \frac{P_{i j}}{1+(i-j)^{2}}
$$

where:

$\mathrm{P}_{\mathrm{ij}}=$ Probability of the co-occurrence of pixel values $\mathrm{i}$ and $\mathrm{j}$ in an image.

$\mathrm{N}$ represents the total number of pixel value co-occurrences in the image.

$\mu=$ Mean of all $(\mathrm{Pij} * \mathrm{i}) \mu=\sum_{i, j=0}^{N-1} i P_{i j}$.

$\sigma^{2}=$ the variance of the intensities of all reference pixels in the relationships that contributed to the GLCM, calculated as: $\sigma^{2}=\sum_{i, j=0}^{N-1} P_{i j}(i-\mu)^{2}$. 


\section{EXPERIMENTAL RESULTS}

The used method was tested on various Knee X-ray images of different age and gender for calculating the joint space thickness needed in the detection of osteoarthritis. This method has been successfully implemented by using MATLAB R2017a.

The input image is shown in Figure. 2 and converted grayscale image is shown in Figure 3.and Figure 4 shows the filtered image. Figures 5-10 shows the contrast-enhanced, threshold, gradient, dilated, cropped and region of interest (ROI) images respectively. The ROI is used to find the thickness which is the distance between the upper and lower cartilages.

The experiment is conducted on $100 \mathrm{Knee} \mathrm{X}$-ray samples and tabulated only 10 results shown in Table 1 . The average thickness of the all images found to be 1.9194. Some cases are having very less cartilage 0.8465 showing severe degeneration of the cartilage and some images having 2.46 indicates healthy Knee.

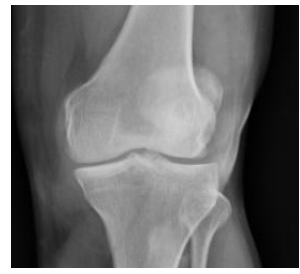

Figure 2. Input Image

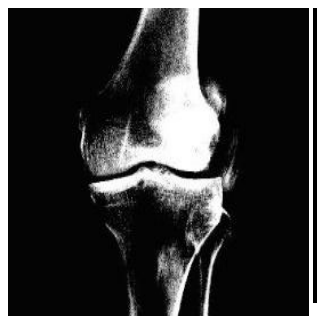

Figure 5. ContrastEnhanced Image

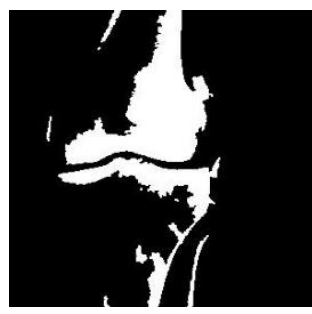

Figure 8. Dilated

Image

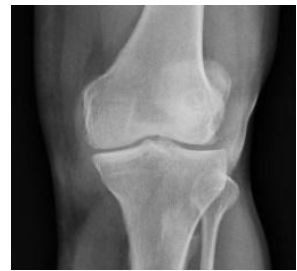

Figure 3. RGB to Gray

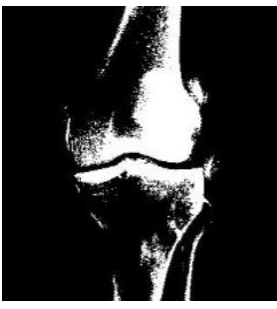

Figure 6. Threshold Image

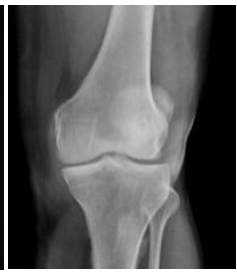

Figure 4. Filtered Image

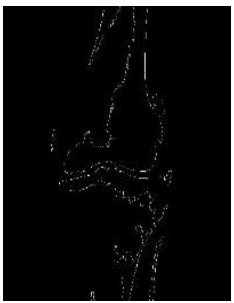

Figure 7.

Binary

Gradient Image

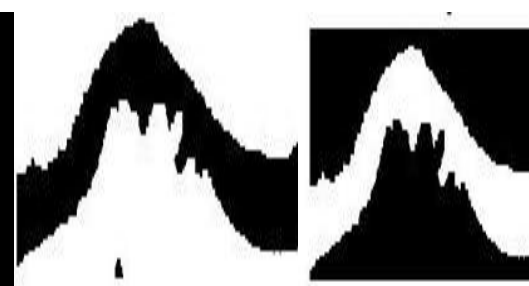
Region of Interest (ROI)
Figure 10. A
Table .1 Joint Space Thickness Measurement Results

\begin{tabular}{|c|c|c|c|c|c|}
\hline $\begin{array}{l}\mathrm{Ca} \\
\mathrm{se}\end{array}$ & $\begin{array}{c}\text { Contr } \\
\text { ast }\end{array}$ & $\begin{array}{c}\text { Correlat } \\
\text { ion }\end{array}$ & Energy & $\begin{array}{c}\text { Homogen } \\
\text { eity }\end{array}$ & $\begin{array}{c}\text { Thicknes } \\
\text { s }\end{array}$ \\
\hline Cas & 262.2 & & & & \\
\hline $\mathrm{e}-1$ & 79 & 0.9754 & 0.6809 & 0.9024 & 1.6164 \\
\hline Cas & 434.2 & & & & \\
\hline$e-2$ & 06 & 0.9604 & 0.6859 & 0.9035 & 2.4626 \\
\hline Cas & 393.1 & & & & \\
\hline$e-3$ & 88 & 0.9650 & 0.7015 & 0.9132 & 2.4648 \\
\hline Cas & 590.9 & & & & \\
\hline$e-4$ & 14 & 0.9563 & 0.5862 & 0.8465 & 1.8505 \\
\hline Cas & 481.5 & & & & \\
\hline$e-5$ & 07 & 0.9579 & 0.6829 & 0.9039 & 1.9898 \\
\hline Cas & 378.6 & & & & \\
\hline$e-6$ & 98 & 0.9781 & 0.6169 & 0.9226 & 0.8760 \\
\hline Cas & 529.1 & & & & \\
\hline$e-7$ & 18 & 0.9669 & 0.5984 & 0.8838 & 2.3941 \\
\hline Cas & 591.6 & & & & \\
\hline$e-8$ & 89 & 0.9621 & 0.5624 & 0.8542 & 1.8795 \\
\hline Cas & 493.1 & & & & \\
\hline$e-9$ & 79 & 0.9563 & 0.6677 & 0.8876 & 1.9021 \\
\hline Cas & & & & & \\
\hline e- & 513.6 & & & & \\
\hline 10 & 87 & 0.9564 & 0.6437 & 0.8772 & 1.7576 \\
\hline $\mathbf{A V}$ & 466.8 & & & & \\
\hline G & 46 & 0.9635 & 0.6433 & 0.8895 & 1.9194 \\
\hline
\end{tabular}

\section{CONCLUSION}

Detection of the cartilage thickness is very important is diagnosing the OA severity. An attempt has been made to simplify the detection of the cartilage part using simple image processing approaches. The proposed method consists of adaptive histogram equalization, thresholding, binary masking and cropping region of interest and GLCM. The proposed method helps to detect the cartilage portion, segment and measure the thickness of the cartilage. Based on the thickness calculated, doctors can take further steps in diagnosing. Automatic segmentation of the cartilage will be the scope of our future work. 


\section{REFERENCES}

[1] Felson D.T, Niu J, Neogi T, Goggins J, Nevitt M .C, Roemer F, Torner J, Lewis C.E, and Guermazi A, 2016. "Synovitis and the risk of knee osteoarthritis: the MOST Study”. Vol 24, Issue 3, pp.458-464, Mar 2016.

[2] Liukkonen M.K, Mononen M.E, Klets O, Arokoski J.P, Saarakkala S, and Korhonen R.K, "Simulation of Subject-Specific Progression of Knee Osteoarthritis and Comparison to Experimental Follow-Up Data: Data from the Osteoarthritis Initiative", Vol 7, Issue 1, pp.9177, Aug 2017.

[3] Jessie Thomson, Terence O’Neill, David Felson, Tim Cootes "Automated Shape \& Texture Analysis for Detection of Osteoarthritis from Radiographs of the Knee", International Conference on Medical Image Computing and Computer-Assisted Intervention pp.127134, Oct 2015.

[4] Shamir L, Ling S.M, Scott Jr William W, Bos A, Orlov N, Macura T.J, Eckley D.M, Ferrucci L, Goldberg I.G, "Knee X-ray image analysis method for automated detection of Osteoarthritis". IEEE Transactions on Biomedical Engineering, Vol 56, Issue 2, pp.407-415, Feb 2009.

[5] Shepherd D.E, and Seedhom B.B, "Thickness of human articular cartilage in joints of the lower limb". Vol 58, Issue 1, pp.27-34, Jan 1999.

[6] Mahima Shanker Pandey, S.S Soam, Surya Prakash Tripathi, "Detection of Knee Osteoarthritis Using XRay", International Journal of Computer Science and Information Technologies, ISSN: 0975-9646, Mar 2016.

[7] Samir K.Bandyopadhyay "An Edge Detection Algorithm for Human Knee Osteoarthritis Images", Journal of Global Research in Computer Science, Vol 2, Issue 4,

[8] Prafull Sharma, Joshua Madhukar Singh “ A Novel Approach towards X-ray Bone Image Segmentation using Discrete Step Algorithm", International Journal of Emerging Trends \& Technology in Computer Science, Vol 2, Issue 5, PP:191-195, ISSN 2278-6856, 2013.

[9] Subromoniam M, Barani S, and Rajini V "A noninvasive computer-aided diagnosis of osteoarthritis from digital X-ray images", Biomedical Research, ISSN 0970938X, Vol 26, Issue 4, pp.721-729, 2015.
[10] Mengko T.L, Wachjudi R.G, Suksmono A.B, Danudirdjo D "Automated Detection of Unimpaired Joint Space for Knee Osteoarthritis Assessment”, IEEE, pp.400-403, June 2005.

[11] Lior Shamir "A Computer analysis method for correlating knee X-ray with continuous indicators", International Journal of Computer Assisted Radiology and Surgery (CARS), Vol 6, pp.699-704, 2011.

[12] Kawathekar P.P and Karande K.J, "Severity analysis of Osteoarthritis of the knee joint from X-ray images: A Literature review", IEEE, pp. 648-652, July 2014.

[13] Cashman P.M, Kitney R.I, Gariba M.A, and Carter M.E, "Automated techniques for visualization and mapping of articular cartilage in MR images of the osteoarthritic knee: a base technique for the assessment of microdamage and submicro damage", Vol 99, Issue 1, pp.42-51, Mar 2002.

[14] Dian Pratiwi, Diaz D.Santika, and Bens Pardamean "An Application of Backpropagation Artificial Neural Network Method for Measuring the Severity of Osteoarthritis", International Journal of Engineering \& Technology, Volume 11, No.3, June 2011.

[15] Emuoyibofarhe O.Justice and Taiwo K.F "Fuzzy-Based system for determining the severity level of knee Osteoarthritis", International Journal of Intelligent Systems and Applications (IJISA), ISSN: 2074-904X, pp. 46-53, 2012.

[16] Gornale S.S, Patravali P.U, and Manza R.R, "A Survey on Exploration and Classification of Osteoarthritis Using Image Processing Techniques". ISSN, 7, pp.2229-5518, June 2016.

[17] N. Otsu. "A threshold selection method from gray level histograms", IEEE Transaction systems Man and Cybernetics, Vol 9, pp.62-66, 1979.systems. Man. and Cybernetics, 9:62-66.

[18] Chan, Tony F, B. Yezrielev Sandberg, and Luminita A. Vese. "Active contours without edges for vector-valued images." Journal of Visual Communication and Image Representation11, Vol 2, pp.30-141, 2000. 